RETRACTION

doi:10.1038/nature09809

\title{
Mediation of pathogen resistance by exudation of antimicrobials from roots
}

Harsh P. Bais, Balakrishnan Prithiviraj, Ajay K. Jha, Frederick M. Ausubel \& Jorge M. Vivanco

Nature 434, 217-221 (2005)

The authors wish to retract this Letter after a key reference by Walker et $a l .{ }^{1}$ (ref. 9 in this Letter) was retracted from the scientific literature. The withdrawn paper $^{1}$ reported ten compounds exuded by Arabidopsis thaliana roots, which were used in this Letter to monitor the defence response in Arabidopsis seedlings. In this Letter, these ten compounds were shown to have antimicrobial activity against specific pathovars of the bacterial phytopathogen Pseudomonas syringae but not against the pathovar Pseudomonas syringae pv. tomato strain DC3000 that is a highly virulent pathogen of Arabidopsis. Moreover, wild-type $P$. syringae DC3000 suppressed the exudation of the ten compounds whereas a DC3000 hrcC mutant did not, leading to the conclusion that DC3000 type III effectors block the exudation or synthesis of the ten compounds. As a consequence of the retraction of the Walker et al. ${ }^{1}$ paper, the validity of the use of the ten compounds as markers of the Arabidopsis defence response is now in doubt. Thus, the data in Fig. 3, Table 1, Supplementary Figs 5-8 and Supplementary Table 1 cannot be used to support the conclusions that $P$. syringae DC3000 is generally resistant to antimicrobial compounds exuded by Arabidopsis or that $P$. syringae DC3000 type III effectors block the exudation or synthesis of antimicrobial compounds.

1. Walker, T. S., Bais, H. P., Halligan, K. M., Stermitz, F. R. \& Vivanco, J. M. Metabolic profiling of root exudates of Arabidopsis thaliana. J. Agric. Food Chem. 51, 2548-2554 (2003). 